Aim of the study: We evaluated the possible effects of comorbid diseases and functional capacity on the survival of elderly female patients with breast cancer.

Material and methods: The study included 159 breast cancer patients aged 65 years or older. Functional status of the patients was evaluated using Katz's index of activities of daily living (ADL) and Lawton and Brody's Instrumental ADL (IADL) scale.

Results: ADL-based evaluation revealed 121 patients $(76.1 \%)$ were independent, 34 (21.4\%) semi-dependent and 4 (2.5\%) dependent whereas IADL-based evaluation showed 69 patients (43.4\%) were independent, 67 patients $(42.1 \%)$ semi-dependent and 23 patients (14.5\%) dependent. Among the patients, 69 (43.4\%) had one comorbid disease, 62 (39.0\%) had two and $26(16.4 \%)$ had three or more. Of the entire cohort, $60.4 \%$ received adjuvant chemotherapy. Based on ADL index, overall survival (OS) was significantly better in semi-dependent and independent patients than in dependent patients $(p=0.001)$. In the upfront non-metastatic patient subgroup, disease-free survival (DFS) was favourable in the independent patients according to $A D L$ index $(p=0.001)$. Having more than one comorbid disease had an unfavourable effect on OS. In the multiple regression analysis of non-metastatic patients, stage, triple-negative histology and ADL index remained significant in terms of OS $(p=0.008$, HR: 3.17 , $\mathrm{Cl}: 1.35-7.44 ; p=0.027, \mathrm{HR}: 2.78, \mathrm{Cl}:$ 1.172-6.91; and $p=0.006, \mathrm{HR}: 0.29, \mathrm{Cl}$ : $0.12-0.70$, respectively).

Conclusions: In elderly patients with breast cancer, evaluation of daily living activities and comorbid diseases are as important as staging and subclassification of breast cancer in the determination of prognosis and survival.

Key words: geriatric, breast, cancer.

Contemp Oncol (Pozn) 2014; 18 (3): 204-210 DOI: $10.5114 /$ wo.2014.42252

\section{Effects of comorbidities and functional living activities on survival in geriatric breast cancer patients}

Ozlem U. Sonmez, Ulku Y. Arslan, Onur Esbah, Kaan Helvaci, Ibrahim Turker, Ummugul Uyeturk, Burcin Budakoglu, Oznur Bal, Berna Oksuzoglu

Ankara Dr AY Oncology Training and Research Hospital, Department of Medical Oncology, Ankara, Turkey

\section{Introduction}

Breast cancer is the most common cancer and the second leading cause of cancer-related deaths among women in the USA. As life expectancy is improving and the geriatric population is growing, ageing becomes an important risk factor for breast cancer [1]. The estimated lifetime risk of a new breast cancer is 1 in 15 for women 70 years or older, 1 in 29 for 60-69 years, 1 in 27 for $40-59$ years, and 1 in 207 for 39 years or younger [2]. Approximately $45 \%$ of patients are 65 years of age or older at the time of initial diagnosis [3]. Age is usually a discriminating factor for chemotherapy preferences. Elderly patients are less likely to be treated according to guidelines or consensus statements. This type of undertreatment decision has caused a lower survival rate of elderly breast cancer patients compared to younger counterparts [4]. In fact, ageing is an individual process and the rate of functional disorders varies among different individuals [5]. Due to comorbidities, pretreatment evaluation only of elderly cancer patients' functional status according to Eastern Cooperative Oncology Group (ECOG) performance status does not appear sufficient for such patients. Presently, an international sole standard method for geriatric assessment does not exist. However, comprehensive geriatric assessment (CGA) consists of comorbidity, feeding, socioeconomic situation, functional status and geriatric syndromes [6]. A complete assessment of elderly patients' health status can be performed with Katz's index of activities of daily living (ADL) and Lawton and Brody's Instrumental ADL (IADL) scale to form a comprehensive geriatric assesment (CGA) [7-9]. In the present study, we determined the impact of CGA and comorbidities on survival and whether breast cancer patients 65 years of age or older receive optimal treatment.

\section{Material and methods}

This study included 159 of the 1014 breast carcinoma patients diagnosed between July 1999 and February 2011 who were 65 years of age or older at the time of diagnosis, and followed-up for more than 6 months. The demographic features of patients, location of tumour, histopathological features, receptor and HER2 status were recorded. If HER2 results were scored as 2, a silver in situ hybridisation (SISH) method was used to determine HER2 status. Consequently, patients whose HER2 score was either 0 or 1 immunohistochemically or SISH-negative were considered negative while patients whose HER2 score was 3 or SISH-positive were considered positive. Implemented surgery, radiotherapy and systemic treatment type, dose and schedule were recorded from patients' charts. 
The existence and numbers of comorbid diseases were reviewed. The ECOG performance status evaluation scale from 0 to 4 was used to evaluate the patients' performance on admission. Evaluation of patients' functional status at the beginning of treatment was conducted using Katz's ADL index [7] and Lawton and Brody's IADL scale [9]. ADL index assesses patients' activities such as dressing, toileting, transferring, continence and feeding. By counting each basic daily life activity separately, the dependency status of a patient is scored. Each patient was given 3 points if able to perform the daily activities independently, 2 points if semi-dependent and 1 point if dependent. According to the ADL index, each activity score is added and the score of 0-6 points indicates dependency, 7-12 semi-dependency and 13-18 independency. The IADL scale includes information related to telephoning, cooking, shopping, daily housework, doing laundry, getting on and off public transportation, using drugs and change money. An individual receives 3 points if able to perform these activities independently, 2 points if receiving some help and 1 point if unable to perform activities without any help. IADL scale describes 0-8 points as dependent, 9-16 as semi-dependent and 17-24 as independent.

\section{Statistical analysis}

The data were analyzed using Statistical Package for Social Sciences (SPSS version 15.0, SPSS Inc. Chicago, IL, USA) software. Disease-free survival (DFS) and overall survival (OS) period and possible factors affecting survival were calculated. DFS was defined as the period between diagnosis and time of first metastasis or death caused by the disease, whereas OS was defined as the period between the time of diagnosis and the date of last control or death. Survival periods were calculated using Kaplan-Meier survival analysis as well as the detection of factors that may affect survival using Cox regression analysis. A $p$-value of $<0.05$ was considered statistically significant.

\section{Results}

The median age of the patients was 71 years (range; 65-91 years). According to ADL, 121 patients (76.1\%) were evaluated as independent, 34 (21.4\%) semi-dependent and 4 (2.5\%) dependent. According to IADL, 69 patients (43.4\%) were considered independent, 67 (42.1\%) semi-dependent and 23 (14.5\%) dependent. Sixty-nine patients (43.4\%) had 1 comorbid disease, 62 (39.0\%) had 2 and 26 (16.4\%) had 3 or more. The most commonly reported comorbid diseases were hypertension, diabetes mellitus, atherosclerosis (and its complications), chronic obstructive pulmonary disease (COPD) and osteoarthrosis.

Six asymptomatic patients (3.8\%) were admitted due to the lesion detected accidentally on screening mammography. Sixteen patients (10.1\%) had at least 1 family member with breast cancer. Clinical and demographic features of the patients are summarised in Table 1.

The most histopathological type was invasive ductal carcinoma (87.4\%). Sixty patients (37.7\%) had grade 3 tumour. ER status was positive (+) in 123 (77.4\%) cases and PR (+) in 105 (66.0\%). The number of triple-negative pa- tients was 17 (11.1\%), HER2-positive was 43 (27.9\%) and HR-positive/HER2-negative was 94 (61\%). Tumour characteristics of the patients are shown in Table 1.

A total of 85 patients (53.4\%) received adjuvant radiotherapy and 96 patients (60.4\%) received adjuvant chemotherapy. Main treatment features of these patients are summarised in Table 2.

One hundred patients (62.9\%) received adjuvant hormonotherapy. Among the hormone-treated patients, 67 (67\%) used aromatase inhibitors (AIS) alone, 23 (23\%) used tamoxifen and 10 (10\%) used AIS and tamoxifen. The median duration of adjuvant hormonotherapy was 25 months (range; 1-96 months).

In the metastatic setting only 19 patients (12\%) received hormonotherapy as the first-line systemic treatment. Of the 25 patients who received chemotherapy as the firstline treatment of metastatic illness, 18 (72.0\%) received combination chemotherapy and 7 (28\%) received monotherapy.

At the median 27-month follow-up (range; 6-139 months), 1-year OS rate for the entire cohort was $97.8 \%$ whereas the 2-year OS rate was $90.5 \%$. The predicted 5 -year OS rate was $75.2 \%$. Survival was not statistically significantly different with respect to the median age $(p=0.58)$.

According to the ADL index of the entire study population, DFS and OS of dependent patients were worse than semi-dependent and independent patients ( $p=0.001$, Fig. 1 ; $p<0.0001$, Fig. 2, respectively). Median OS of dependent patients was 12 months (range; 7.5-16.4 months), while median OS of semi-dependent and independent patients could not be determined.

Survival analysis according to the ADL index for the non-metastatic subgroup at the time of diagnosis showed that median DFS for the dependent group was 12 months, whereas median DFS of semi-dependent and independent patients could not be not determined $(p<0.001$, Fig. 3). Median OS time of the dependent patients was 12 months, and median OS of semi-dependent and independent patients could not be determined. Median OS of the dependent patients was worse than the other patients $(p<0.001)$.

Disease free survival and OS were not statistically significantly different between the entire study population and the upfront non-metastatic group at the time of the diagnosis according to the IADL $(p>0.005)$.

The survival analysis of the study population according to the number of comorbid diseases showed an inverse relation meaning OS was decreasing while the number of comorbid diseases was increasing $(p=0.02)$ but DFS did not change $(p=0.085)$.

The survival analysis of upfront non-metastatic patients according to the number of comorbid diseases showed that DFS was decreasing while comorbid diseases increased ( $p=0.044)$, and OS was also decreasing but was not statistically significant ( $p=0.054$ ).

In the Cox regression multivariant analysis, only ADL was an independent prognostic factor for DFS ( $p=0.001$, $H R=0.30)$. When evaluated for non-metastatic patient subgroup, ADL remained an important prognostic indica- 
Table 1. Clinical - demographic and tumor characteristics of geriatric age patients with breast cancer

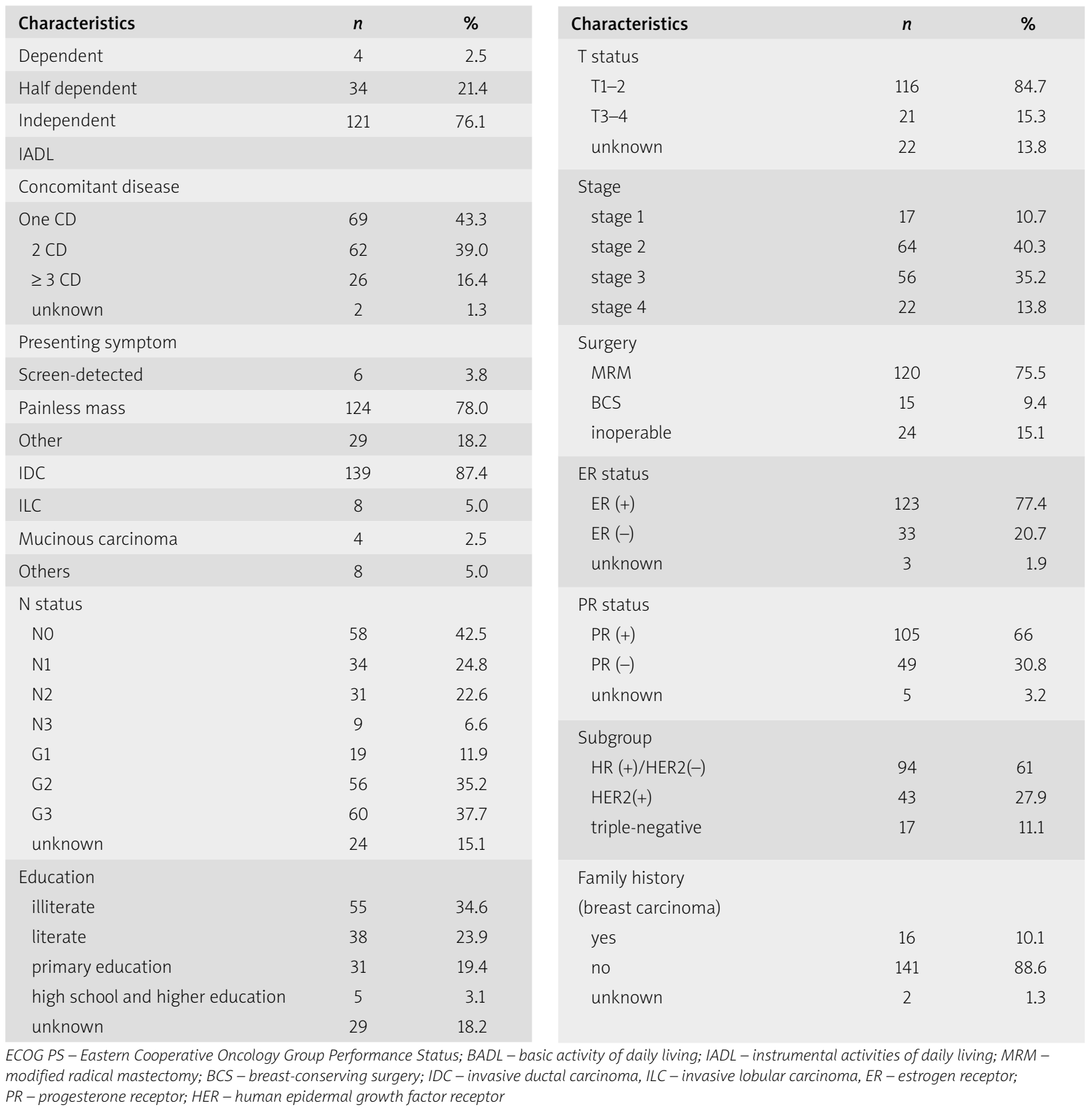

tor $(p=0.04, \mathrm{HR}=0.30)$, as well as stage $(p=0.21, \mathrm{HR}=$ $=2.67)$ and subtype of breast cancer $(p=0.004, \mathrm{HR}=3.09)$ for OS.

\section{Discussion}

Due to improved life conditions, the number of elderly people in the overall population is increasing in Turkey. Ageing is an important risk factor in cancer development, due to many reasons such as age-related molecular changes, immune system deficiency and increased sensitivity of the aging tissues to carcinogens [5]. As observed with other types of cancer, the incidence of breast cancer also increases with ageing [10]. According to SEER data, more than half of cancer cases are 65 years of age or older [11]. In this study, the percentage of patients aged 65 years and older was $15.6 \%$ among all breast cancer patients. The lower percentage may be explained by inadequate cancer screening and awareness as well as difficulties of elderly people to reach medical services due to deficiency of social assistance and economical reasons. Education has a significant influence on overall survival. Patients with the highest educational status also lived longer [12]. According to USA cancer statistics reported in 2010, the rate of the elderly breast cancer patients who were admitted with upfront metastases at the time of diagnosis was $6 \%$ [13], whereas in a study conducted in Turkey, 59\% of the patients with metastatic breast cancer at the time of diagnosis were over the age of 50 years [14]. Furthermore, because $35 \%$ of our study population was illiterate and approximately $86 \%$ were poorly educated, $14 \%$ of the pa- 
Table 2. Basic features of treatment of geriatric breast cancer patients

\begin{tabular}{|c|c|c|}
\hline Radiation therapy & $n$ & $\%$ \\
\hline Post MRM & 73 & 45.9 \\
\hline Post BCS & 14 & 8.8 \\
\hline \multicolumn{3}{|l|}{ Palliative RT } \\
\hline yes & 18 & 11.3 \\
\hline no & 54 & 34 \\
\hline \multicolumn{3}{|l|}{ Adjuvant RT } \\
\hline yes & 85 & 53.4 \\
\hline no & 74 & 46.6 \\
\hline \multicolumn{3}{|l|}{ Adjuvant chemotherapy } \\
\hline yes & 96 & 60.4 \\
\hline no & 63 & 39.6 \\
\hline \multicolumn{3}{|c|}{ Adjuvant treatment duration } \\
\hline less than 12 weeks & 25 & 28.7 \\
\hline more than 12 weeks & 62 & 71.3 \\
\hline \multicolumn{3}{|c|}{ Adjuvant anthracycline status } \\
\hline received & 83 & 86.5 \\
\hline did not receive & 13 & 13.5 \\
\hline \multicolumn{3}{|c|}{ Anthracycline preference } \\
\hline epirubisin & 24 & 28.9 \\
\hline adriamisin & 59 & 71.1 \\
\hline
\end{tabular}

\begin{tabular}{|lcc|}
\hline Adjuvant chemotherapy denial reason & $n$ & $\%$ \\
\hline Early stage & 10 & 15.9 \\
\hline Performance status bad & 5 & 7.9 \\
\hline Advanced age & 8 & 12.7 \\
\hline Patient refused chemotherapy & 13 & 20.6 \\
\hline Received neoadjuvant & 5 & 7.9 \\
\hline Up front metastatic & 22 & 34.9 \\
\hline Adjuvant taxane status & & \\
\hline received & 47 & 49.5 \\
\hline did not receive & 48 & 50.5 \\
\hline Taxane preference & & \\
\hline paclitaxel & 12 & 22.2 \\
\hline docetaxel & 42 & 77.8 \\
\hline Adjuvant anthracycline + taxane status & & \\
\hline received & 23 & 42 \\
\hline did not receive & 54 & 56.3 \\
\hline received & & 46.5 \\
\hline did not receive & & \\
\hline
\end{tabular}

MRM - modified radical mastectomy; BCS - breast conservative surgery

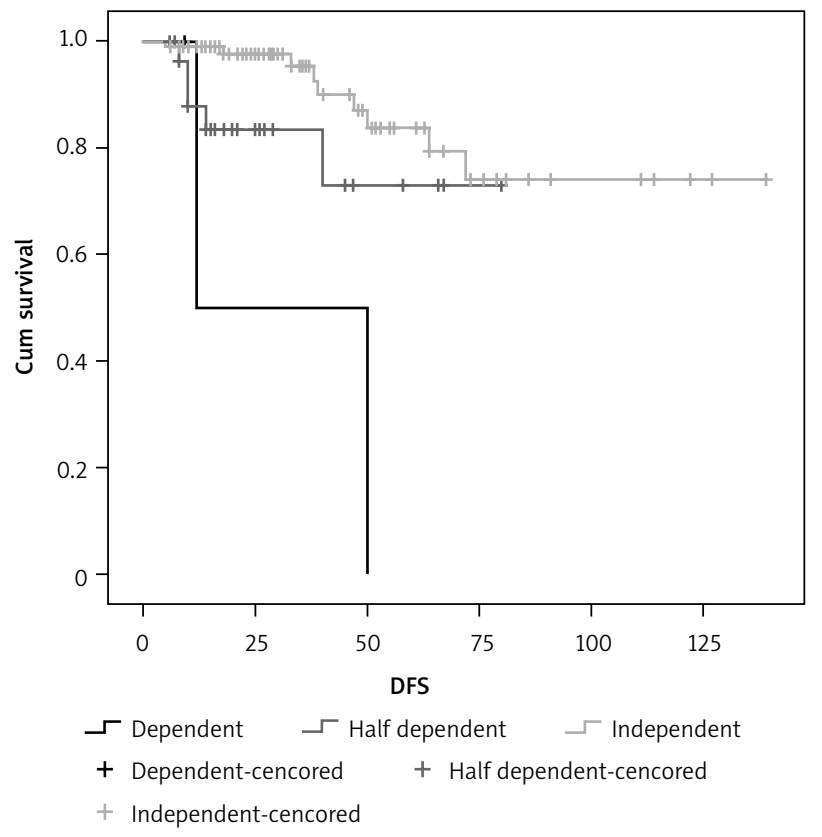

Fig. 1. Disease free survival (DFS) according to the ADL scale, for whole group $(p=0.001)$

tients admitted were in a metastatic stage at the time of diagnosis. When the presenting complaints of the patients were evaluated, the detection by screening mammography was observed to be as low as 3.8\%. Another study in our country, mammography screening was observed 3.9\%

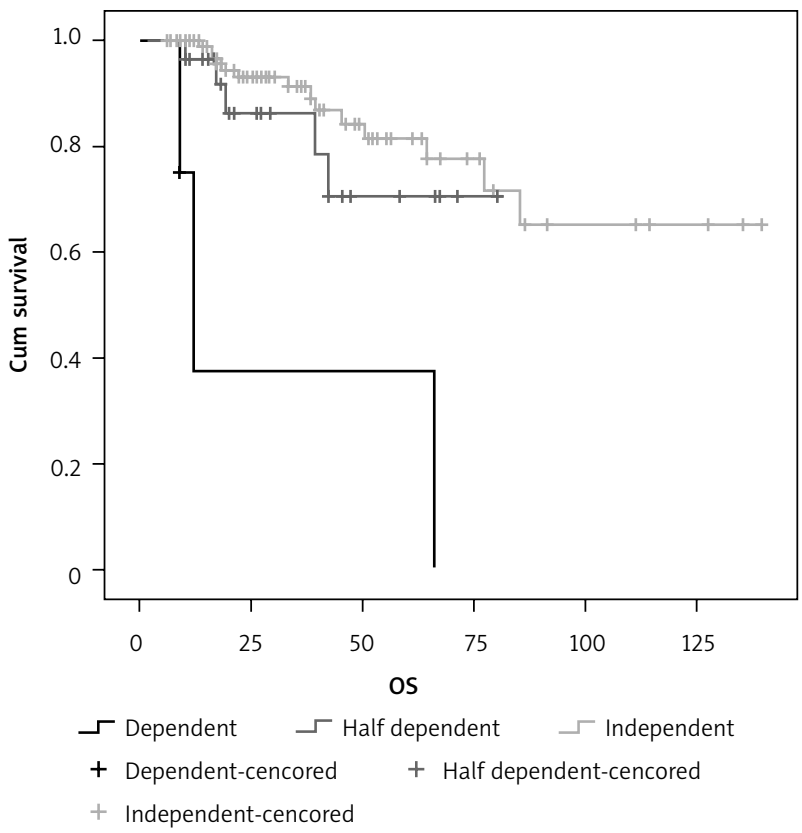

Fig. 2. Overall survival (OS) according to the ADL scale, for whole group $(p<0.0001)$

[15]. While the presence of breast cancer in a first-degree relative increases the risk by at least 2 -fold, the presence of breast cancer in two first-degree relatives increases the risk by 4 - to 6 -fold. When the familial history of breast cancer was evaluated in the present study, approximate- 


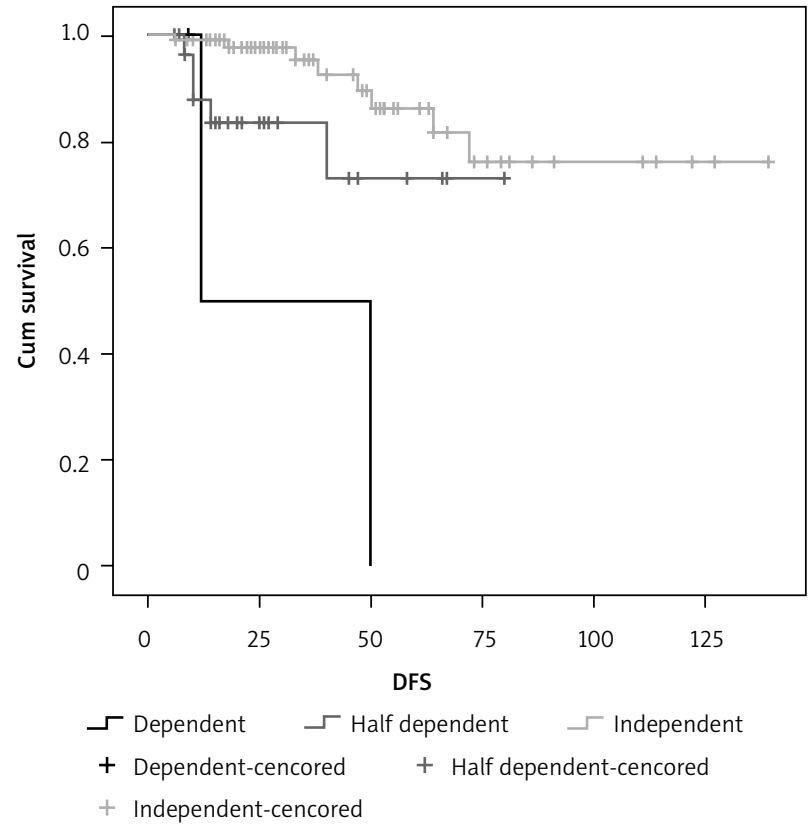

Fig. 3. Disease free survival (DFS) according to the ADL scale, for upfront non-metastatic subgroup $(p=0.001)$

ly $10.1 \%$ of the patients had a familial history of breast cancer, consistent with the literature $[16,17]$ and 19\% had a history of cancer other than breast cancer in their family.

The majority of elderly patients with cancer die due to comorbid diseases rather than cancer. Elderly breast cancer patients usually have one or more pre-existing comorbid diseases such as heart diseases, COPD, hypertension, osteoarthrosis, diabetes mellitus, and atherosclerosis $[18,19]$. Similar to the literature, $43.3 \%$ of the patients had 1 comorbid disease, $39 \%$ had 2 and $16.4 \%$ had 3 or more comorbid diseases in our study. Most commonly observed comorbid diseases included hypertension, diabetes mellitus, atherosclerosis (and its complications), COPD and osteoarthrosis. A Danish study confirmed that comorbid conditions are an independent adverse factor for death after breast cancer [20]. As expected, our study showed that for the entire study population, the presence of more than one comorbid disease had a statistically significant unfavourable effect on OS. For DFS, although not statistically significant, there was an inverse correlation between the number of comorbid diseases and DFS. Evaluation of chronological age is not an appropriate approach to estimate the individual life expectancy and to make therapeutic decisions. Physical function is also an important factor that affects survival. Evaluation of elderly patients with cancer by ECOG performance status is mostly inadequate because of the comorbid diseases that are present in the majority of these patients.

It is possible to use CGA to evaluate the complete health status of elderly patients. CGA is considered to be more efficient for the care and treatment of elderly patients in addition to standard medical evaluation, and consensus guidelines recommend CGA for elderly patients [21-24]. Therapeutic management of elderly patients mainly consists of the evaluation of functional status, social assistance, psychological status, nutritional status, medications used and the diseases that affect morbidity [22]. The conditions that may hinder cancer treatment may be evaluated by considering factors such as comprehensive geriatric evaluation, comorbid disease, nutrition, polypharmacy, early dementia, caregiver abuse, support, transportation, administration of the financial issues and shopping. Therapeutic and supportive strategies should be determined and appropriate symptom management may be ensured by classifying patients as dependent, semi-dependent or independent. The patients who are dependent on any type of help for ADL and who have poor social assistance have the highest risk for encountering chemotherapy-related complications. Cancer therapy complications may be mitigated by determining the medical problems with previously undetermined CGA results, ADL and IADL may be rapidly performed in an outpatient clinic setting, and may provide independent information regarding mortality and morbidity [7-9]. In a study where the scoring was based on bathing ability, distance walked and the ability to pull-push objects by an elderly person, functional morbidity index was evaluated according to physical functions of 4516 patients aged 70 years or older [25]. This report demonstrated that patients who lost one third of their functions had a survival less than 2 years, whereas patients with excellent functions had a lower mortality risk. Approximately $25 \%$ of the patients aged 65 years or older need assistance to meet the criteria of ADL or IADL. Fifty percent of the people aged 85 years or older need assistance to meet the criteria of basic ADL (BADL). The guidelines recommend performing functional evaluations in all elderly patients [26]. In a study conducted in Turkey, ADLs of people aged 60 years or older who have been living in a house or in an institution were evaluated and more than half of the patients were independent in their ADLs and semi-dependent in their IADLs [27]. In our study, ADL-based evaluation showed $76.1 \%$ of the patients were independent, $21.4 \%$ were semi-dependent and 2.5\% were dependent, whereas IADL-based evaluation showed $43.4 \%$ of the patients were independent, $41.5 \%$ were semi-dependent and $13.8 \%$ were dependent. When the patients were compared in terms of OS and were found to be dependent, semi-dependent or independent based on ADL scoring of the study population, median OS was 12 months for dependent patients and ADL-based dependency had a statistically significant effect on OS, which was also the case when patients with metastatic disease were excluded from the analysis. When an IADL-based evaluation was performed for all groups, despite the observation of worse OS in dependent patients, median survival times could not be determined in each of the three groups due to the short follow-up period. In patients with non-metastatic disease, although not statistically significant, there was a trend towards improved OS in independent patients. Similarly, DFS results favoured significantly independent patients in terms of ADL and IADL. Surgical approach was not different between elderly and younger breast cancer patients [28], thus our results were compatible with the literature.

In this study, age was not considered a differentiating factor in radiation therapy choices. Although clinicians 
administer adjuvant chemotherapy to many patients with breast cancer, they tend to give less chemotherapy to patients aged 65 years or older because geriatric patients have a high percentage of comorbid diseases that may interact with the therapy; the therapy is not well tolerated due to ageing process and limited clinical data are available for this age group [29]. In a UK survey that investigated factors involved in decisions regarding chemotherapy in HER2-positive early-stage breast cancers, comorbid diseases were considered by $34 \%$ and age by $25 \%$ of clinicians [1]. In our study, $60.4 \%$ of the patients were administered adjuvant chemotherapy. Because chemotherapy was not administered due to advanced age in only 15.9\% of the patients, a low percentage compared to the literature [1], age was a less important determinant in decisions regarding chemotherapy in our centre. However, febrile neutropenia was detected in approximately $14.7 \%$ and the need for dose reduction in 15\% of the patients indicating that advanced pre-treatment examination is indispensable in elderly patients with breast cancer. EORTC [30] and NCCN [21] Guidelines recommend the primary prophylactic use of granulocyte colony-stimulating factor (G-CSF) in cancer patients aged 65 years or older. In another study on patients with metastatic breast cancer (MBC) aged 65 years or older, doxorubicin-containing regimens were as effective as in younger patients and acute side effects and cardiotoxicity did not differ from younger patients [31]. In a prospective study in which age-related toxicities were pharmacologically evaluated in the patients with breast cancer treated with doxorubicin and cyclophosphamide, advanced age was not a contraindication for adjuvant therapy for breast cancer and a significant correlation was not demonstrated between age and effects such as neutropenia, cardiac dysfunction and decrease in quality of life [32]. The number of geriatric cancer patients is increasing incrementally and will become a more important health problem in the future. A substantial part of these patients will need therapeutic agents with a high potential for side effects, such as triple-negative or HER2+ adjuvant chemotherapy and trastuzumab.

Studies have suggested that therapeutic regimens used in young patients may also be used in medically fit elderly patients. However, evaluation of performance status has been emphasised to prevent severe side effects. Age should not be the only differentiating factor in the decision regarding therapy. This study demonstrated that functional ADL and comorbid diseases were as effective as tumour biology on OS. The evaluation to determine the functional status of elderly patients may be conducted within a short period of time in outpatient oncology clinics. Accordingly, a better determination can be made of which patient will or will not tolerate chemotherapy and the appropriate therapy can be administered.

The authors declare no conflict of interest.

\section{References}

1. Ring A.The influences of age and co-morbidities on treatment decisions for patients with Her2 positive early breast cancer. Crit Rev Oncol Hematol 2010; 76: 127-32.

2. Siegel R, Ward E, Brawley O, Jemal A. Cancer statistics, 2011: the impact eliminating socioeconomic and racial disparities on premature cancer deaths. CA Cancer J Clin 2011; 61: 212-36.

3. De Santis C, Siegel R, Bandi P, Jemal A. Breast cancer statistics, 2011. CA Cancer J Clin 2011; 61: 409-18.

4. Owusu C, Hurria A, Muss H. Adjuvant Therapy for Older Women with Early-Stage Breast Cancer: Treatment Selection in a Complex Population American Society of Clinical Oncology. In Educational Book; 48 ${ }^{\text {th }}$ Annual meeting June 1-5, 2012 Chicago, Illinois.

5. Tuna S. Kanserli geriatrik hastalarda komorbidite ve klinik değerlendirme. Türk Onkoloji Dergisi 2007; 22: 192-6 [In Turkish].

6. Pallis AG, Fortpied C, Wedding U, et al. EORTC elderly task force position paper: approach to the older cancer patient. Eur J Cancer 2010; 46: 1502-13.

7. Katz S, Downs TD, Crash H, Grotz RC. Progress in development of the index of ADL. Gerontologist 1970; 10: 20-30.

8. Shelkey M, Wallace M. Katz. Index of independence in activities of daily living. J Gerontol Nurs 1999; 25: 8-9.

9. Lawton MP, Brody EM. Assessment of older people: self-maintaining and instrumental activities of daily living. Gerontologist 1969; 9: $179-86$.

10. Wildiers H, Kunkler I, Biganzoli L, et al. Management of breast cancer in elderly individuals: recommendations of the International Society of Geriatric Oncology. Lancet Oncol 2007; 8: 1101-15.

11. National Cancer institute. Cancer Statistics Review 1975-2005. Surveillance, Epidemiology and End Results (SEER) Program, April 26, 2010.

12. Nowara E, Suwinski R. Impact of educational differences as measure of socioeconomic status on survival for breast cancer patients. Wspolczesna Onkol 2012; 16: 345-9.

13. Balducci L, Exterman M, Carreca I. Manegemant of Breast cancer in the older women. Cancer Control 2011; 8: 431-41.

14. Sezgin C, Zekioğlu O, Gökmen E. Illk tanıda metastatik meme kanseri ile başvuran hastaların sağkalımını etkileyen prognostik faktörler. Türk Onkoloji Dergisi. 2006; 21: 20-25 [In Turkish].

15. Uyeturk U, Budakoglu B, Turker I, et al. Assesment of general characteristics of patients with primary metastatic breast carcinoma: single center experience. Contemp Oncol (Pozn) 2013; 17: 450-55.

16. Garber J. Breast cancer. In: Risk Factors. Silva EO, Zumda S (eds.). $3^{\text {rd }}$ ed. Elsevier Saunders, Oxford 2005; 26-53.

17. Barisic A, Glendon G, Weerasooriya N, Andrulis IL, Knight JA. Accuracy of Self-Reported Breast Cancer Information among Women from the Ontario Site of the Breast Cancer Family Registry. J Cancer Epidemiol 2012; 2012: 310804.

18. Yancik R, Wesley MN, Ries LA, Havlik RJ, Edwards BK, Yates JW. Effect of age and comorbidity in postmenopausal breast cancer patients aged 55 years and older. JAMA 2001; 285: 885-92.

19. Yancik R, Havlik RJ, Wesley MN, Ries L, Long S, Rossi WK, Edwards BK. Cancer and comorbidity in older patients: a descriptive profile. Ann Epidemiol 1996; 6: 399-412.

20. Land LH, Dalton SO, Jensen MB, Ewertz M. Influence of comorbidity on the effect of adjuvant treatment and age in patients with early-stage breast cancer. Br J Cancer 2012; 107: 1901-7.

21. National Comprehensive Cancer Network (NCCN) Guidelines Version 1. 2013.

22. Extermann M, Hurria A. Comprehensive geriatric assessment for older patients with cancer. J Clin Oncol 2007; 25: 1824-31.

23. Hurria A, Naeim A, Elkin E, Limaye S, Grover A, Hudis C, Pearce C, Robson M. Adjuvant treatment recommendations in older women with breast cancer: a survey of oncologists. Crit Rev Oncol Hematol 2007; 61: 255-60.

24. Green FL. American Joint Committee on Cancer (AJCC). Cancer Staging Manual, $6^{\text {th }}$ ed. Green FL, Page DL, Fleming ID et al. (eds.). Springer-Verlag, New York 2002. Appendix 1.

25. Walter LC, Brand RJ, Counsell SR, Palmer RM, Landefeld CS, Fortinsky $\mathrm{RH}$, Covinsky KE. Development and validation of a prognostic index for 1-year mortality in older adults after hospitalization. JAMA 2001; 285: 2987-94. 
26. Kristjanson SR, Wyller TB. Introduction. Section 1. In: Schrijvers D, Aopro M, Zakotnik B, Audisio R, Van Haltren H, Hurria A. Esmo Handbook of Cancer in the Senior Patient. First published. London and New York: Informa Health Care 2010; 1-7.

27. Tel H, Sabancıoğulları S. Evde ve kurumda yaşayan 60 yaş ve üzeri bireylerin günlük yaşam aktivitelerini sürdürme ve yalnızlık yaşama durumu. Turkish Journal of Geriatrics 2006; 9: 34-4 [In Turkish].

28. Biganzoli L. Breast cancer in the senior patient. Section 12. In: Schrijvers D, Aopro M, Zakotnik B, Audisio R, Van Haltren H, Hurria A. Esmo Handbook of Cancer in the Senior Patient. First published. Informa Health Care, London, New York 2010; 92-7.

29. Mandelblatt JS, Sheppard VB, Hurria A, et al. Breast cancer adjuvant chemotherapy decisions in older women: The role of patient preference and interactions with physicians. J Clin Oncol 2010; 28: 19; 3146-53.

30. Repetto L, Biganzoli L, Koehne CH, Luebbe AS, Soubeyran P, Tjan-Heijnen VC, Aapro MS. EORTC Cancer in the Elderly Task Force guidelines for the use of colony-stimulating factors in elderly patients with cancer. Eur J Cancer 2003; 39: 2264-72.

31. Ibrahim NK, Frye DK, Buzdar AU, Walters RS, Hortobagyi GN. Doxorubicin-based chemotherapy in elderly patients with metastatic breast cancer. Tolerance and outcome. Arch Intern Med 1996; 22 882-8.

32. Dees EC, O'Reilly S, Goodman SN, et al. A prospective pharmacologic evaluation of age related toxicity of adjuvant chemotherapy in women with breast cancer. Cancer Invest 2000; 18: 521-9.

\section{Address for correspondence}

Ozlem Uysal Sonmez MD

Department of Medical Oncology

Ankara Dr AY Oncology Training and Research Hospital

Mehmet Akif Ersoy Mahallesi

13.Cad. No:56 Demetevler/Yenimahalle

06200 Ankara, Turkey

e-mail:dr.ozlemus@gmail.com

Submitted: 3.04 .2014

Accepted: $\quad 4.04 .2014$ 